\title{
Valve Hemodynamics and Clinical Outcomes After Transcatheter Aortic Valve Replacement for a Small Aortic Annulus
}

\author{
Norihiko Kamioka, ${ }^{1}$ MD, Takeshi Arita, ${ }^{1}$ MD, Michiya Hanyu, ${ }^{2}$ MD, Masaomi Hayashi, ${ }^{1}$ MD, \\ Shun Watanabe, ${ }^{2} \mathrm{MD}$, Shiro Miura, ${ }^{1} \mathrm{MD}$, Akihiro Isotani, ${ }^{1} \mathrm{MD}$, Yoshio Arai, ${ }^{2} \mathrm{MD}$, \\ Shinichi Kakumoto, ${ }^{3}$ MD, Kenji Ando, ${ }^{1}$ MD and Shinichi Shirai, ${ }^{1}$ MD
}

\begin{abstract}
Summary
The hemodynamics and outcomes in patients with a small aortic annulus (SAA) after transcatheter aortic valve replacement (TAVR) with a second-generation transcatheter heart valve remain unclear. We investigated whether TAVR with a Sapien XT (Edwards Lifesciences, Irvine, California) influences postprocedural valve hemodynamics and long-term outcome in high-risk SAA patients compared with surgical aortic valve replacement (SAVR).

We retrospectively identified 94 SAA patients who underwent aortic valve replacement $($ TAVR $=35$ and SAVR $=59$ ). SAA was defined as an aortic annulus diameter $\leq 20 \mathrm{~mm}$, measured by preprocedural transesophageal echocardiography.

The mean age was 80.2 years. The mean Society of Thoracic Surgeons-Predicted Risk of Mortality was $6.8 \%$. The post-procedural peak transvalvular velocity and mean pressure gradient were significantly lower in the TAVR cohort than in the SAVR cohort, whereas the postprocedural aortic valve area was significantly higher in the TAVR cohort. Severe prosthesis-patient mismatch (PPM) occurred less frequently after TAVR than SAVR (TAVR $2.9 \%$ versus SAVR $22.0 \%, P=0.01$ ). The two-year mortality in SAA patients was similar between the two groups.

TAVR with a Sapien XT in SAA patients improved the valve hemodynamics and reduced the incidence of PPM compared with SAVR. TAVR patients had a similar 2-year mortality despite higher risk baseline characteristics. To avoid PPM and the consequent poor outcomes, TAVR can be considered an alternative option to SAVR in high surgical risk patients with SAA.
\end{abstract}

(Int Heart J 2019; 60: 86-92)

Key words: Balloon-expandable valve, TAVR, TAVI, SAVR, Prosthesis-patient mismatch

A small aortic annulus (SAA) has been reported to not only increase the incidence of prosthesispatient mismatch (PPM) but also contribute to worse procedural and clinical outcomes following surgical aortic valve replacement (SAVR). ${ }^{1-3)}$ Transcatheter aortic valve replacement (TAVR) using a first-generation transcatheter heart valve (THV) was reported to improve hemodynamics, reduce PPM, and improve clinical outcomes compared with SAVR, even in patients with SAA. $^{4-7)}$ However, the hemodynamics and outcomes in SAA patients after TAVR with a second-generation THV remain unclear. Therefore, we investigated whether TAVR with a second-generation THV influences valve hemodynamics and long-term clinical outcomes in high-risk SAA patients compared with SAVR.

\begin{abstract}
Methods
From January 2013 to December 2014, 291 consecutive patients who underwent their first aortic valve replacement for severe aortic stenosis at our institution were retrospectively enrolled. All patients were reviewed by our local multidisciplinary heart team who determined the appropriate procedure for each patient as per their preprocedural risk. All patients who were assigned with TAVR were deemed as high surgical risk due to high STS risk score, advanced age, frailty, and severe comorbidity. From this cohort, 165 cases were excluded as their aortic annulus was $>20 \mathrm{~mm}$ by preprocedural transesophageal echocardiography (TEE). Of the remaining 126 cases, we included patients with a preprocedural peak transvalvular velocity $(\mathrm{PV}) \geq 4.0 \mathrm{~m} / \mathrm{second}$ or a mean pressure gradient (MPG) $\geq 40 \mathrm{mmHg}$ who then received a bioprosthetic valve by TAVR or SAVR. In the SAVR group, we also
\end{abstract}

From the ${ }^{1}$ Department of Cardiology, Kokura Memorial Hospital, Fukuoka, Japan, ${ }^{2}$ Department of Cardiovascular Surgery, Kokura Memorial Hospital, Fukuoka, Japan and ${ }^{3}$ Department of Anesthesiology, Kokura Memorial Hospital, Fukuoka, Japan.

Address for correspondence: Shinichi Shirai, MD, Department of Cardiology, Kokura Memorial Hospital, 3-2-1 Asano, Kokurakita, Kitakyushu 802-8555, Fukuoka, Japan. E-mail: shirai440130@gmail.com

Received for publication November 28, 2017. Revised and accepted April 13, 2018.

Released in advance online on J-STAGE November 20, 2018.

doi: 10.1536/ihj.17-656

All rights reserved by the International Heart Journal Association. 
excluded 4 patients who received a mechanical prosthetic valve: a Regent aortic valve $(n=3)$ (St. Jude Medical, St. Paul, Minnesota) and a Carbomedics aortic valve $(n=1)$ (Sorin, Milan, Italy). All the patients agreed to participate in this study and provided written informed consent. This study was approved by the local institutional review board.

All TAVR procedures were performed in accordance with the standard procedure of TAVR under general anesthesia in a hybrid operating room. ${ }^{8)}$ The access route and size of a prosthetic valve were determined in each patient based on a preprocedural assessment, including multidetector computed tomography, echocardiography, Doppler ultrasound of the peripheral vessels, and angiography. The transapical approach was selected because of small lumen size, significant calcification, or extensive tortuosity of the iliofemoral arterial access. All the TAVR procedures were performed using a second-generation THV device, a Sapien XT valve system (Edwards Lifesciences, Irvine, California).

The SAVR procedures were performed via standard median sternotomy with a cardiopulmonary bypass. In this study, we used two types of surgical bioprostheses: a Carpentier-Edwards prosthesis Magna Ease valve (Edwards Lifesciences) and a Mosaic Ultra valve (Medtronic, Minneapolis, Minnesota). Operators determined the valve type prior to the procedure. Native aortic valve and perivalvular calcification were carefully resected in all the SAVR cases before implantation of the prosthesis. The size of the bioprosthesis was determined by calibrating to proprietary valve sizers. Fifty-seven of the valves were implanted in the supra-annular position. The remaining two valves were implanted in the intra-annular position. No patients underwent an aortic annular enlargement or an aortic root replacement despite SAA.

The aortic annulus diameter was measured preoperatively between the hinge points of the leaflet in midsystole from the long-axis view using TEE, according to the guidelines of the American Society of Echocardiography. ${ }^{9)}$ The left ventricular and valvular functions were assessed pre- and postprocedure with transthoracic echocardiography according to the established guidelines. ${ }^{9-11)}$ The aortic valve area (AVA) postprocedure was calculated according to the guideline and indexed for the patient's body surface area. ${ }^{11)}$ PPM was defined as an indexed AVA $<0.65 \mathrm{~cm}^{2} / \mathrm{m}^{2}$.

The aim of this study was to compare the valve hemodynamics and clinical outcomes of SAA patients after TAVR using a Sapien XT valve with those after SAVR using a conventional stented bioprosthesis. We first compared valve hemodynamics, including the incidence of PPM, and then compared postprocedure outcomes between the two groups. The primary endpoint was 2-year mortality, and the secondary endpoint was 30-day mortality.

The patients' baseline characteristics were included in our assessment. Coronary artery disease was defined as any of the following: angina pectoris with documented coronary artery stenosis, previous myocardial infarction, or previous coronary artery revascularization. Cerebrovascular disease was defined as transient ischemic attack or ischemic stroke. Renal failure was defined as a serum creatinine level $>1.5 \mathrm{mg} / \mathrm{dL}$. Outcomes and complications were reported in accordance with the definition by the valve academic research consortium (VARC)-2 criteria. ${ }^{12}$

Clinical status and valve hemodynamics were evaluated at baseline, at discharge, at 1, 3, and 6 months, and annually thereafter. All the information was collected from the medical record or direct phone contact with patients or their families.

Statistical analyses were performed using JMP version 10.0.2 (SAS Institute, Cary, North Carolina). Quantitative values are reported as the mean \pm standard deviation, and qualitative values are reported as numbers and percentages. Continuous variables were examined using the unpaired $t$-test or nonparametric analysis by the Wilcoxon rank sum test. Categorical variables were compared using the chi-square test or Fisher's exact test. Survival curves were analyzed using the Kaplan-Meier method and compared with the log-rank test. $P$-values less than 0.05 were considered to be statistically significant.

\section{Results}

We identified 94 patients $($ TAVR $=35$ and SAVR $=$ 59) who met our inclusion criteria. Sapien XT 23-mm valves were used for all TAVRs. The median-adjusted contrast volume to deploy a valve was $-1.0 \mathrm{~mL}$ (interquartile range: -1.0 to $0.0 \mathrm{~mL}$ ). In the SAVR group, 35 Mosaic ultravalves $(19-\mathrm{mm}$ valve $=32$ and $21-\mathrm{mm}$ valve = 3) and 24 Carpentier-Edwards prosthesis Magna ease valves $(19-\mathrm{mm}$ valve $=15$ and $21-\mathrm{mm}$ valve $=9)$ were used. The mean age and mean Society of Thoracic Surgeons-Predicted Risk of Mortality of the entire cohort were 80.2 years and $6.8 \%$, respectively. Unsurprisingly, TAVR patients were older and were more likely to have a history of coronary artery bypass grafting or balloon aortic valvuloplasty than SAVR patients (Table I). Additionally, TAVR patients exhibited more severe symptoms of heart failure and higher logistic European System for Cardiac Operative Risk Evaluation (EuroSCORE). SAVR patients were more likely to have diabetes mellitus. The baseline valve hemodynamics were similar for the PV, MPG, and AVA between the two groups (Table II). Some of the SAVR patients underwent planned concomitant procedures: coronary artery bypass grafting (17 cases), mitral valve replacement (4 cases), and combined mitral and tricuspid valve replacement ( 1 case).

The postprocedural PV and MPG were significantly lower in the TAVR cohort than in the SAVR cohort, and the postprocedural AVA was significantly larger in the TAVR cohort. PPM was observed in only 1 case $(2.9 \%)$ post-TAVR, whereas it occurred in 13 cases $(22.0 \%, P=$ 0.01 ) post-SAVR (Figure 1). Significant paravalvular leakage (PVL) was observed in 4 patients post-TAVR (Table III). The superiority in valve hemodynamics after TAVR with a Sapien XT was maintained 6 months after the procedure (Figure 1A-C).

The mean follow-up was $20.6 \pm 9.3$ months (range: 2.0-38.7 months). The main clinical outcomes are listed in Table III. Procedure success was achieved in all the cases. No procedure-related death was observed. Three patients 
Table I. Baseline Clinical Characteristics

\begin{tabular}{lcccc}
\hline \multicolumn{1}{c}{ Variable } & Overall $(n=94)$ & TAVR $(n=35)$ & SAVR $(n=59)$ & $P$ value \\
\hline Age $($ years $)$ & $80.2 \pm 8.6$ & $85.1 \pm 6.0$ & $77.4 \pm 8.7$ & $<0.001$ \\
Female & $88(93.6 \%)$ & $32(91.4 \%)$ & $56(94.9 \%)$ & 0.67 \\
BMI $\left(\mathrm{kg} / \mathrm{m}^{2}\right)$ & $22.3 \pm 3.8$ & $21.6 \pm 3.4$ & $22.7 \pm 4.0$ & 0.16 \\
BSA $\left(\mathrm{m}^{2}\right)$ & $1.38 \pm 0.14$ & $1.37 \pm 0.17$ & $1.38 \pm 0.13$ & 0.64 \\
NYHA class III/IV & $37(39.4 \%)$ & $19(54.3 \%)$ & $18(30.5 \%)$ & 0.03 \\
Logistic EuroSCORE $(\%)$ & $14.3 \pm 10.2$ & $18.9 \pm 12.8$ & $11.6 \pm 7.3$ & 0.01 \\
EuroSCORE II $(\%)$ & $5.5 \pm 5.7$ & $6.4 \pm 4.7$ & $5.0 \pm 6.1$ & 0.23 \\
STS-PROM $(\%)$ & $6.8 \pm 4.3$ & $6.7 \pm 3.0$ & $6.8 \pm 5.0$ & 0.91 \\
Hypertension & $68(72.3 \%)$ & $26(74.3 \%)$ & $42(71.2 \%)$ & 0.81 \\
Dyslipidemia & $44(46.8 \%)$ & $16(45.7 \%)$ & $28(47.5 \%)$ & 1.00 \\
Diabetes mellitus & $33(35.1 \%)$ & $6(17.1 \%)$ & $27(45.8 \%)$ & 0.01 \\
Renal failure* & $10(10.6 \%)$ & $3(8.6 \%)$ & $7(11.9 \%)$ & 0.74 \\
eGFR (mL/minute/1.73 $\left.\mathrm{m}^{2}\right)$ & $52.3 \pm 23.7$ & $50.9 \pm 21.1$ & $53.1 \pm 25.2$ & 0.50 \\
Smoking habit & $7(7.5 \%)$ & $1(2.9 \%)$ & $6(10.2 \%)$ & 0.25 \\
Cerebrovascular disease & $10(10.6 \%)$ & $1(2.9 \%)$ & $9(15.3 \%)$ & 0.08 \\
Coronary artery disease & $29(30.9 \%)$ & $14(40.0 \%)$ & $15(25.4 \%)$ & 0.17 \\
History of CABG & $4(4.3 \%)$ & $4(11.4 \%)$ & $0(0.0 \%)$ & 0.02 \\
History of PCI & $23(24.5 \%)$ & $10(28.6 \%)$ & $13(22.0 \%)$ & 0.62 \\
History of heart failure & $35(37.2 \%)$ & $10(28.6 \%)$ & $25(42.4 \%)$ & 0.20 \\
History of BAV & $23(24.5 \%)$ & $18(51.4 \%)$ & $5(8.5 \%)$ & $<0.001$ \\
\hline
\end{tabular}

*Renal failure is defined as a serum-creatinine level more than $1.5 \mathrm{mg} / \mathrm{dL}$. Values are expressed mean $\pm \mathrm{SD}$, median (interquartile range) or $n(\%)$. BAV indicates balloon aortic valvuloplasty; BMI, body mass index; BSA, body surface area; CABG, coronary artery bypass grafting; eGFR, estimated glomerular filtration rate; EuroSCORE, European System for Cardiac Operative Risk Evaluation; NYHA, New York Heart Association; PCI, percutaneous coronary intervention; SAVR, surgical aortic valve replacement; SD, standard deviation; STS-PROM, Society of Thoracic Surgery-Predicted Risk of Mortality; and TAVR, transcatheter aortic valve replacement.

Table II. Baseline Echocardiographic Characteristics

\begin{tabular}{lcccc}
\hline \multicolumn{1}{c}{ Variable } & Overall $(n=94)$ & TAVR $(n=35)$ & SAVR $(n=59)$ & $P$ value \\
\hline Peak velocity $(\mathrm{m} / \mathrm{second})$ & $5.08 \pm 0.71$ & $5.21 \pm 0.77$ & $5.01 \pm 0.68$ & 0.19 \\
Mean PG $(\mathrm{mmHg})$ & $60.9 \pm 19.1$ & $64.6 \pm 20.1$ & $58.7 \pm 18.3$ & 0.15 \\
Aortic valve area $\left(\mathrm{cm}^{2}\right)$ & $0.57 \pm 0.13$ & $0.56 \pm 0.11$ & $0.58 \pm 0.13$ & 0.34 \\
Aortic annulus $(\mathrm{mm}) *$ & $19.1 \pm 0.6$ & $19.1 \pm 0.7$ & $19.1 \pm 0.6$ & 0.77 \\
AR $(\geq$ moderate) & $29(30.1 \%)$ & $14(40.0 \%)$ & $15(25.4 \%)$ & 0.14 \\
MR $(\geq$ moderate) & $35(37.2 \%)$ & $10(28.6 \%)$ & $25(42.4 \%)$ & 0.20 \\
TR $(\geq$ moderate $)$ & $21(22.3 \%)$ & $8(22.9 \%)$ & $13(22.0 \%)$ & 0.93 \\
LVEF $(\%)$ & $60.8 \pm 8.9$ & $60.9 \pm 7.8$ & $60.6 \pm 9.5$ & 0.88 \\
SV $(\mathrm{mL})$ & $71.2 \pm 14.7$ & $72.6 \pm 13.2$ & $70.4 \pm 15.7$ & 0.49 \\
SI $\left(\mathrm{mL} / \mathrm{m}^{2}\right)$ & $52.1 \pm 10.4$ & $53.8 \pm 9.6$ & $51.1 \pm 10.8$ & 0.23 \\
LV mass index $\left(\mathrm{g} / \mathrm{m}^{2}\right)$ & $137.0 \pm 37.5$ & $135.1 \pm 39.1$ & $138.1 \pm 36.7$ & 0.71 \\
\hline
\end{tabular}

*Aortic annulus diameter was measured by transesophageal echocardiography. AR indicates aortic regurgitation; LV, left ventricle; LVEF, left ventricle ejection fraction; MR, mitral regurgitation; PG, pressure gradient; SAVR, surgical aortic valve replacement; SI, stroke volume index; SV, stroke volume; TAVR, transcatheter aortic valve replacement; and TR, tricuspid regurgitation.

had a stroke after TAVR. In addition to the complications listed in Table III, 1 patient suffered coronary obstruction immediately after TAVR and underwent percutaneous coronary intervention as a bail-out procedure. There was no 30-day mortality in either cohort. The Kaplan-Meier curve revealed similar mortality at 2 years in both groups (TAVR $19.4 \%$ versus SAVR $19.2 \%, P=0.95$, Figure 2).

\section{Discussion}

This study demonstrated two major findings: (1) the valve hemodynamics were significantly better after TAVR with a Sapien XT than after SAVR with a conventional stented valve; (2) SAA patients after TAVR achieved similar long-term clinical outcomes compared with patients after SAVR despite the higher risk baseline characteristics in the TAVR cohort. Therefore, TAVR using a Sapien XT can be considered an alternative to SAVR in SAA patients with high surgical risk.

In this study, TAVR with a Sapien XT demonstrated superior valve hemodynamics and a lower incidence of PPM than SAVR, which was consistent with previous 
A Peak velocity

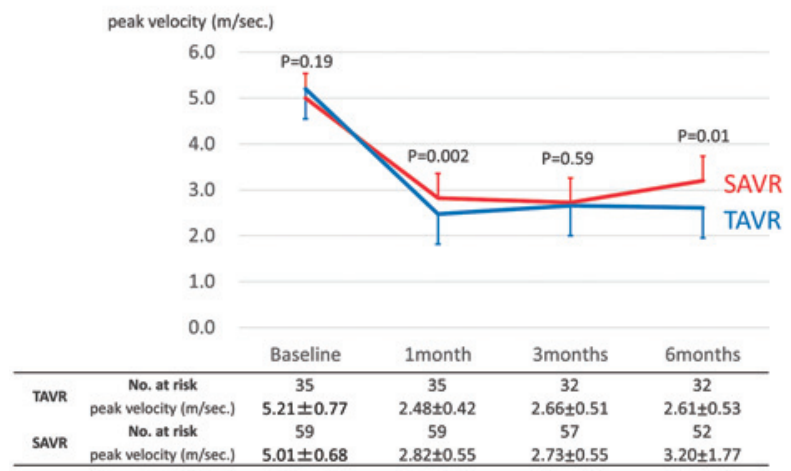

C Aortic valve area

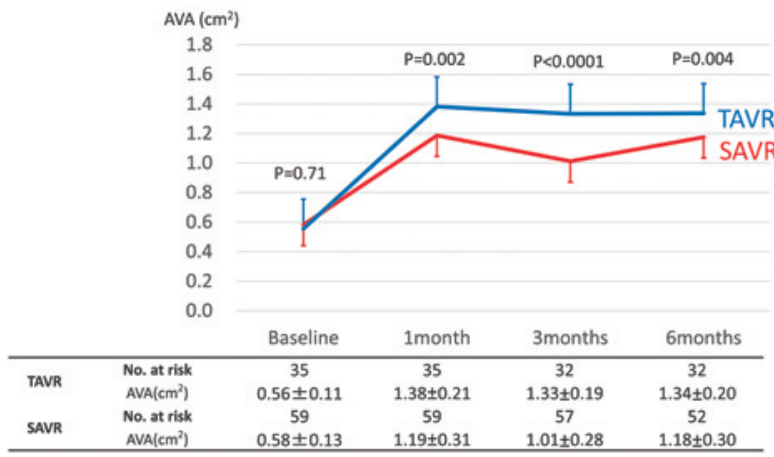

B Mean pressure gradient

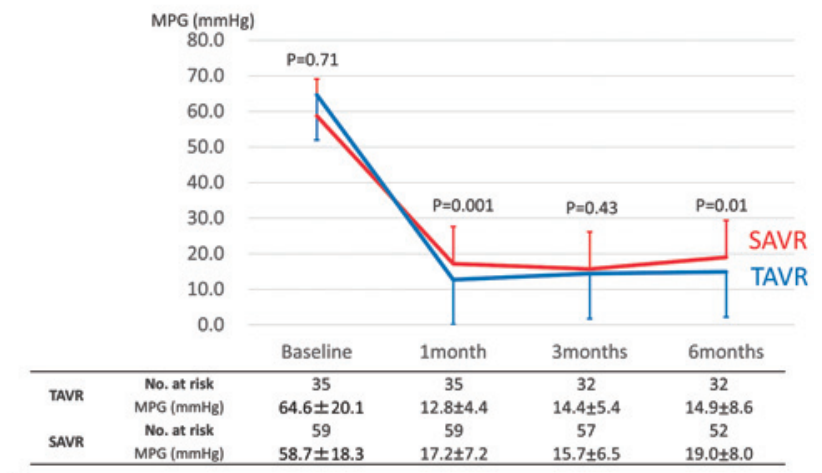

D Prosthesis-Patient Mismatch

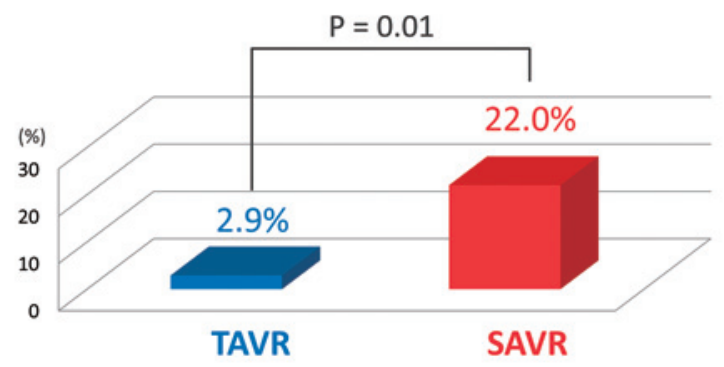

Figure 1. Valve hemodynamics. A: Change of peak velocity. B: Change of mean pressure gradient. C: Change of aortic valve area. D: Incidence of PPM. PPM was defined as an indexed AVA $<0.65 \mathrm{~cm}^{2} / \mathrm{m}^{2}$. AVA indicates aortic valve area; MPG, mean pressure gradient; PPM, prosthesispatient mismatch; SAVR, surgical aortic valve replacement; and TAVR, transcatheter aortic valve replacement.

studies.,6) These hemodynamic findings were preserved until 6 months postprocedure. To avoid PPM and the consequent adverse long-term effects in SAA patients who underwent SAVR, supra-annular valve implantation was performed in most of our SAVR cohort. ${ }^{13)}$ Despite the use of this procedure, PPM was observed more frequently after SAVR in this study. Importantly, the incidence of severe PPM after SAVR in this study $(22.0 \%)$ was lower than in previous studies (28.1-33.9\%), and the incidence of severe PPM after TAVR was also lower in this study (2.2\%) than in previous studies $(7.0-19.7 \%){ }^{4,6,14)}$ The low incidence of PPM post-TAVR may be due to our unique procedural strategy. We implanted a $23-\mathrm{mm}$ valve with underfilled volume for all the SAA patients because a 23$\mathrm{mm}$ valve was the smallest valve available during the study period. Further investigation is required to determine the long-term impact of implanting a valve with underfilled volume in SAA.

In addition, the structural differences between the THV and the surgical bioprosthesis (i.e., the valvular membrane sewn in the thin strut stent [cobalt chrome metal] versus the thick stent frame [fabric-covered wire frame]) and the difference in the manner of implantation (i.e., hanging with dilatation of the aortic annulus [the oversizing concept] versus sewing inside of the aortic annulus) may affect the postprocedural AVA. ${ }^{15-17)}$ In SAA patients, our study demonstrates that the structure of THV may contribute to a larger AVA than surgical valves at the time of the procedure. In addition, the structural difference between THVs and surgical valves may favor THVs when considering a valve-in-valve procedure for the degenerative valve in the future. ${ }^{17)}$ Such differences may influence the long-term outcomes and require further investigation.

SAA has been reported to contribute to a high incidence of PPM, leading to worse procedural and clinical outcomes following SAVR. ${ }^{1-3,18)}$ Prior randomized studies have also shown the impact of aortic annulus size on outcomes following TAVR, particularly in SAA patients. ${ }^{4,19}$ However, this SAA study demonstrated similar long-term outcomes after both TAVR and SAVR. This difference could be attributed to higher age and higher surgical risk in the TAVR patients than in the SAVR patients. Moreover, previous reports revealed the impact of PPM in larger populations and at longer time periods than our study. ${ }^{20-22)}$ Therefore, further studies with larger populations and longer observational periods are required to investigate the impact of PPM on long-term outcome in SAA patients.

The incidence of stroke was reported to be similar after SAVR and TAVR, although potential sources of embolization differ between the two procedures. ${ }^{23,24)}$ In this study, three patients experienced stroke symptoms after TAVR using a Sapien XT, although all the symptoms 
Table III. Clinical Outcomes

\begin{tabular}{lcccc}
\hline & Overall $(n=94)$ & TAVR $(n=35)$ & SAVR $(n=59)$ & $P$ value \\
\hline In-hospital outcomes & & & & \\
All vascular complication & $7(7.5 \%)$ & $4(11.4 \%)$ & $3(5.1 \%)$ & 0.42 \\
$\quad$ Major vascular complication & $3(3.2 \%)$ & $1(2.9 \%)$ & $2(3.4 \%)$ & 1.00 \\
All bleeding & $59(62.3 \%)$ & $23(65.7 \%)$ & $36(61.0 \%)$ & 0.65 \\
$\quad$ LT bleeding & $25(26.6 \%)$ & $1(2.9 \%)$ & $24(40.7 \%)$ & $<0.001$ \\
Major bleeding & $25(26.6 \%)$ & $15(42.9 \%)$ & $10(17.0 \%)$ & 0.01 \\
LT and major bleeding & $50(53.2 \%)$ & $16(45.7 \%)$ & $34(57.6 \%)$ & 0.26 \\
Stroke & $3(3.2 \%)$ & $3(8.6 \%)$ & $0(0.0 \%)$ & 0.05 \\
All AKI & $10(9.6 \%)$ & $3(8.6 \%)$ & $7(11.9 \%)$ & 0.74 \\
AKI $\geq$ stage 2 & $3(3.2 \%)$ & $1(2.9 \%)$ & $2(3.4 \%)$ & 1.00 \\
New PM implantation & $4(4.3 \%)$ & $2(5.7 \%)$ & $2(3.4 \%)$ & 0.63 \\
Echocardiographic outcomes & & & & \\
Mean APG $<20$ mmHg & $76(80.6 \%)$ & $32(91.4 \%)$ & $44(74.6 \%)$ & 0.04 \\
Moderate/severe PVL & $4(4.3 \%)$ & $4(4.3 \%)$ & $0(0.0 \%)$ & 0.02 \\
LVEF $(\%)$ & $60.8 \pm 8.9$ & $60.9 \pm 7.8$ & $60.6 \pm 9.5$ & 0.88 \\
Short- and long-term outcomes & & & & \\
30-day mortality & 0 & 0 & 0 & - \\
1-year mortality & $8(8.5 \%)$ & $4(11.4 \%)$ & $4(6.8 \%)$ & 0.43 \\
2-year mortality & $15(16.0 \%)$ & $6(17.1 \%)$ & $9(15.3 \%)$ & 0.81 \\
\hline AKI
\end{tabular}

AKI indicates acute kidney injury; APG, aortic pressure gradient; LT, life-threatening; LVEF, left ventricular ejection fraction; PM, pacemaker; PVL, paravalvular leakage; SAVR, surgical aortic valve replacement; and TAVR, transcatheter aortic valve replacement.

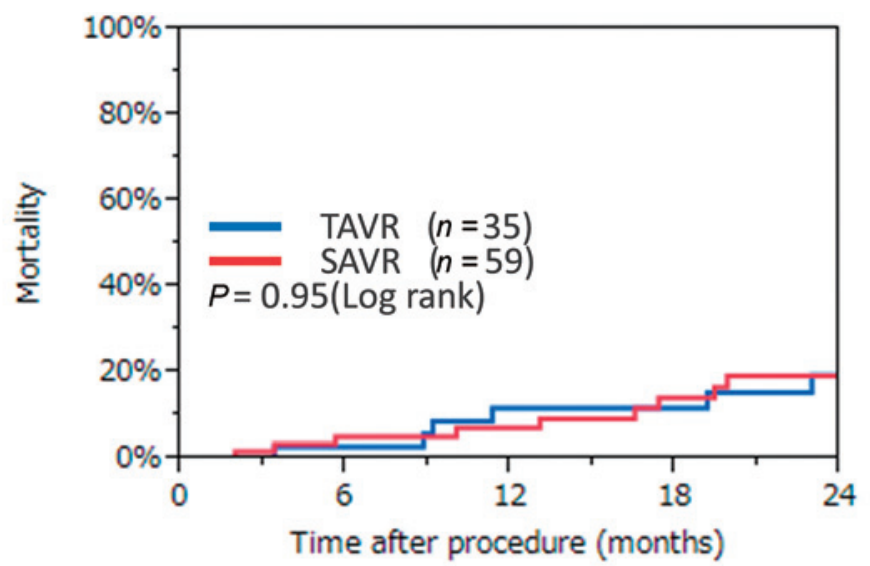

\begin{tabular}{ccccccc}
\hline & Months & 0 & 6 & 12 & 18 & 24 \\
\hline \multirow{4}{*}{ TAVR } & No. at risk & 35 & 35 & 29 & 25 & 20 \\
& $\%$ & 0 & 2.8 & 11.7 & 11.7 & 19.4 \\
& SE & 0 & 0.03 & 0.06 & 0.06 & 0.07 \\
\multirow{4}{*}{ SAVR } & No. at risk & 59 & 56 & 45 & 35 & 16 \\
& $\%$ & 0 & 5.1 & 7.1 & 14.1 & 19.2 \\
& SE & 0 & 0.03 & 0.03 & 0.05 & 0.06 \\
\hline
\end{tabular}

Figure 2. Kaplan-Meier curve of mortality. SAVR indicates surgical aortic valve replacement; and TAVR, transcatheter aortic valve replacement.

were not severe (dizziness, double vision, and visual field defect, respectively). Next-generation valves (i.e., Sapien 3 ) and utilization of stroke prevention devices may help to prevent stroke. ${ }^{25-27)}$

Several limitations could affect our outcomes. First, this was a single-center, retrospective analysis. Therefore, the small sample size may affect our results. Second, we hired the SAA criteria defined by two-dimensional TEE findings according to the report which revealed efficacy of a first-generation THV in SAA patients. ${ }^{5)}$ Aortic annulus diameters measured by multidetector computed tomography or magnetic resonance imaging would have provided more precise information as we commonly use multimodal imaging for the preprocedural planning of structural heart disease. Although the most convenient and reliable way of measuring an aortic annulus diameter remains unclear, the most acceptable SAA definition would be preferred to determine the influence of SAA on the very 
long-term outcomes. Third, a Sapien XT 23-mm valve was used in all the TAVR procedures because it was the smallest commercially available THV during the study period. Nevertheless, no aortic annulus rupture was observed, demonstrating the procedural safety of TAVR with a Sapien XT for SAA patients. In the SAVR group, we used two types of stented bioprostheses. Other newer bioprostheses, such as stentless valves or sutureless valves, may affect the outcomes. ${ }^{28)}$ The difference in bleeding following TAVR and SAVR was not assessed. ${ }^{29-31)}$ Fourth, aortic annular enlargement or root replacement was not performed in our elderly cohort, although some reports have demonstrated efficacy in young patients. ${ }^{3,32,33)}$ The indication of these procedures should be discussed on a case-by-case basis as the adjunctive procedure could make the surgery more complex. ${ }^{34)}$ Finally, the influence of PVL was not considered because its incidence was relatively low in this study, although PVL has been reported to be associated with poor outcome. ${ }^{35-37)}$

\section{Conclusion}

In SAA patients with high surgical risk, TAVR with a Sapien XT exhibited better valve hemodynamics and a lower incidence of PPM than SAVR. In addition, the long-term mortality in SAA patients was similar between the two groups despite the increased age and higher surgical risk in the TAVR population. TAVR can be considered as an alternative to SAVR in high surgical risk SAA patients who are susceptible to PPM and consequent poor clinical outcomes.

\section{Acknowledgment}

The authors thank Frank Corrigan, MD and Hope Caughron, BA, from Emory University Hospital, for help with editing the manuscript and Hiromi Umeda, from the Department of Physiological Examination, Kokura Memorial Hospital, for help with the outstanding record of transthoracic echocardiography.

\section{Disclosures}

Conflicts of interest: Shinichi Shirai, MD is a proctor for transfemoral-TAVR for Edwards Lifesciences. Yoshio Arai, $\mathrm{MD}$ is a proctor for transapical-TAVR for Edwards Lifesciences. The other authors have stated that they have no relationships relevant to the contents of this paper to disclose.

\section{References}

1. Pibarot P, Dumesnil JG. Hemodynamic and clinical impact of prosthesis-patient mismatch in the aortic valve position and its prevention. J Am Coll Cardiol 2000; 36: 1131-41.

2. Pibarot P, Dumesnil JG. Prosthesis-patient mismatch: definition, clinical impact, and prevention. Heart 2006; 92: 1022-9.

3. Dhareshwar J, Sundt TM, Dearani JA, Schaff HV, Cook DJ, Orszulak TA. Aortic root enlargement: What are the operative risks? J Thorac Cardiovasc Surg 2007; 134: 916-24.

4. Pibarot P, Weissman NJ, Stewart WJ, et al. Incidence and se- quelae of prosthesis-patient mismatch in transcatheter versus surgical valve replacement in high-risk patients with severe aortic stenosis: A PARTNER trial cohort-a analysis. J Am Coll Cardiol 2014; 64: 1323-34.

5. Kalavrouziotis D, Rodés-Cabau J, Bagur R, et al. Transcatheter aortic valve implantation in patients with severe aortic stenosis and small aortic annulus. J Am Coll Cardiol 2011; 58: 1016-24.

6. Clavel MA, Webb JG, Pibarot P, et al. Comparison of the Hemodynamic performance of percutaneous and surgical bioprostheses for the treatment of severe aortic stenosis. J Am Coll Cardiol 2009; 53: 1883-91.

7. Hahn RT, Pibarot P, Stewart WJ, et al. Comparison of transcatheter and surgical aortic valve replacement in severe aortic stenosis: A longitudinal study of echocardiography parameters in cohort a of the PARTNER trial (Placement of aortic transcatheter valves). J Am Coll Cardiol 2013; 61: 2514-21.

8. Holmes DR, MacK MJ, Kaul S, et al. 2012 ACCF/AATS/SCAI/ STS expert consensus document on transcatheter aortic valve replacement. J Am Coll Cardiol 2012; 59: 1200-54.

9. Lang RM, Badano LP, Mor-Avi V, et al. Recommendations for cardiac chamber quantification by echocardiography in adults: An update from the American society of echocardiography and the European association of cardiovascular imaging. Eur Heart $\mathrm{J}$ Cardiovasc Imaging 2015; 16: 233-71.

10. Baumgartner H, Hung J, Bermejo J, et al. Echocardiographic Assessment of Valve Stenosis: EAE/ASE Recommendations for Clinical Practice. J Am Soc Echocardiogr 2009; 22: 1-23.

11. Zoghbi WA, Enriquez-Sarano M, Foster E, et al. Recommendations for evaluation of the severity of native valvular regurgitation with two-dimensional and Doppler echocardiography. J Am Soc Echocardiogr 2003; 16: 777-802.

12. Kappetein AP, Head SJ, Généreux P, et al. Updated standardized endpoint definitions for transcatheter aortic valve implantation : the Valve Academic Research Consortium-2 consensus document. Eur Heart J 2012; 33: 2403-18.

13. Botzenhardt F, Eichinger WB, Bleiziffer S, et al. Hemodynamic comparison of bioprostheses for complete supra-annular position in patients with small aortic annulus. J Am Coll Cardiol 2005; 45: 2054-60.

14. Thyregod HGH, Steinbrüchel DA, Ihlemann N, et al. No clinical effect of prosthesis-patient mismatch after transcatheter versus surgical aortic valve replacement in intermediate- and low-risk patients with severe aortic valve stenosis at mid-term follow-up: An analysis from the NOTION trial. Eur J Cardio-thoracic Surg 2016; 50: 721-8.

15. Walther T, Falk V. Hemodynamic evaluation of heart valve prostheses. Paradigm Shift for transcatheter valves? J Am Coll Cardiol 2009; 53: 1892-3.

16. Pibarot P, Dumesnil JG. Prosthetic heart valves: Selection of the optimal prosthesis and long-term management. Circulation 2009; 119: 1034-48.

17. Bapat VN, Attia R, Thomas M. Effect of valve design on the stent internal diameter of a bioprosthetic valve: A concept of true internal diameter and its implications for the valve-in-valve procedure. JACC Cardiovasc Interv 2014; 7: 115-27.

18. Head SJ, Mokhles MM, Osnabrugge RLJ, et al. The impact of prosthesispatient mismatch on long-term survival after aortic valve replacement: A systematic review and meta-analysis of 34 observational studies comprising 27186 patients with 133141 patient-years. Eur Heart J 2012; 33: 1518-29.

19. Rodes-Cabau J, Pibarot P, Suri RM, et al. Impact of Aortic Annulus Size on Valve Hemodynamics and Clinical Outcomes After Transcatheter and Surgical Aortic Valve Replacement: Insights From the PARTNER Trial. Circ Cardiovasc Interv 2014; 7: 701-11.

20. Tasca G, Mhagna Z, Perotti S, et al. Impact of prosthesis-patient mismatch on cardiac events and midterm mortality after aortic valve replacement in patients with pure aortic stenosis. Circulation 2006; 113: 570-6.

21. Mohty-Echahidi D, Malouf JF, Girard SE, et al. Impact of 
prosthesis-patient mismatch on long-term survival in patients with small St Jude medical mechanical prostheses in the aortic position. Circulation 2006; 113: 420-6.

22. Milano AD, De Carlo M, Mecozzi G, et al. Clinical outcome in patients with 19-mm and 21-mm St. Jude aortic prostheses: Comparison at long-term follow-up 2002; 73: 37-43.

23. Mack MJ, Leon MB, Smith CR, et al. 5-year outcomes of transcatheter aortic valve replacement or surgical aortic valve replacement for high surgical risk patients with aortic stenosis (PARTNER 1): a randomised controlled trial. Lancet 2015; 385: 2477-84

24. Daneault B, Kirtane AJ, Kodali SK, et al. Stroke associated with surgical and transcatheter treatment of aortic stenosis: A comprehensive review. J Am Coll Cardiol 2011; 58: 2143-50.

25. Tummala R, Banerjee K, Sankaramangalam K, et al. Clinical and procedural outcomes with the SAPIEN 3 versus the SAPIEN XT prosthetic valves in transcatheter aortic valve replacement: A systematic review and meta-analysis. Catheter Cardiovasc Interv 2017.

26. Seeger J, Gonska B, Otto M, Rottbauer W, Wöhrle J. Cerebral embolic protection during transcatheter aortic valve replacement significantly reduces death and stroke compared with unprotected procedures. JACC Cardiovasc Interv 2017; 10: 2297-303.

27. Rodés-Cabau J, Kahlert P, Neumann FJ, et al. Feasibility and exploratory efficacy evaluation of the embrella embolic deflector system for the prevention of cerebral emboli in patients undergoing transcatheter aortic valve replacement: The PROTAVIC pilot study. JACC Cardiovasc Interv 2014; 7: 1146-55.

28. Santarpino G, Pfeiffer S, Jessl J, et al. Sutureless replacement versus transcatheter valve implantation in aortic valve stenosis: A propensity-matched analysis of 2 strategies in high-risk patients. J Thorac Cardiovasc Surg 2014; 147: 561-7.

29. Kochman J, Rymuza B, Huczek Z, et al. Incidence, Predictors and Impact of Severe Periprocedural Bleeding According to VARC-2 Criteria on 1-Year Clinical Outcomes in Patients After Transcatheter Aortic Valve Implantation. Int Heart J 2016; 57: 35-40.

30. Kleczynski P, Dziewierz A, Bagienski M, et al. Association between blood transfusions and 12-month mortality after transcatheter aortic valve implantation. Int Heart J 2017; 58: 50-5.

31. Généreux P, Cohen DJ, Williams MR, et al. Bleeding complications after surgical aortic valve replacement compared with transcatheter aortic valve replacement: Insights from the PARTNER i trial (Placement of Aortic Transcatheter Valve). J Am Coll Cardiol 2014; 63: 1100-9.

32. Kulik A, Al-Saigh M, Chan V, et al. Enlargement of the small aortic root during aortic valve replacement: is there a benefit? Ann Thorac Surg 2008; 85: 94-100.

33. Sommers KE, David TE. Aortic valve replacement with patch enlargement of the aortic annulus. Ann Thorac Surg 1997; 63: 1608-12.

34. Dayan V, Vignolo G, Soca G, Paganini JJ, Brusich D, Pibarot P. Predictors and outcomes of prosthesis-patient mismatch after aortic valve replacement. JACC Cardiovasc Imaging 2016; 9: 924-33.

35. Hayashida K, Lefèvre T, Chevalier B, et al. Impact of postprocedural aortic regurgitation on mortality after transcatheter aortic valve implantation. JACC Cardiovasc Interv 2012; 5: 1247-56.

36. Sponga S, Perron J, Dagenais F, et al. Impact of residual regurgitation after aortic valve replacement. Eur $\mathrm{J}$ Cardio-thoracic Surg 2012; 42: 486-92.

37. Détaint D, Lepage L, Himbert D, et al. Determinants of Significant paravalvular regurgitation after transcatheter aortic valve implantation. JACC Cardiovasc Interv 2009; 2: 821-7. 